\title{
Exploration and Research on Animal Model of Brain Schistosomiasis
}

\author{
Shengbin Ma1,2, Fei Yang ${ }^{1}$, Mingcan $\mathrm{Wu}^{1,2^{*}}$ \\ ${ }^{1}$ Health Science Center, Yangtze University, Jingzhou, China \\ ${ }^{2}$ Department of Orthopedic, The First School of Clinical Medicine \& The First Hospital of Jingzhou, Yangtze University, Jingzhou, \\ China \\ Email: 411826541@qq.com, 773916832@qq.com, *wumingcan@yangtzeu.edu.cn
}

How to cite this paper: Ma, S.B., Yang, F. and $\mathrm{Wu}$, M.C. (2020) Exploration and Research on Animal Model of Brain Schistosomiasis. Yangtze Medicine, 4, 302-309. https://doi.org/10.4236/ym.2020.44028

Received: December 18, 2019 Accepted: September 27, 2020 Published: September 30, 2020

Copyright $\odot 2020$ by author(s) and Scientific Research Publishing Inc. This work is licensed under the Creative Commons Attribution International License (CC BY 4.0).

http://creativecommons.org/licenses/by/4.0/

\begin{abstract}
Objective: A rabbit model of schistosoma granuloma was established by injecting Schistosoma japonicum eggs via carotid artery after opening blood-brain barrier with mannitol. Methods: Rabbits in the experimental group were injected with $1 \mathrm{ml}$ of Schistosoma japonicum egg suspension after injecting mannitol into carotid for $20 \mathrm{~min}$ to establish a rabbit model; The rabbits in the negative control group were injected with mannitol through carotid artery for $20 \mathrm{~min}$, followed by $1 \mathrm{ml}$ normal saline injection; rabbits in the positive control group were injected with $1 \mathrm{ml}$ suspension of Schistosoma japonicum eggs after coning cranial. The clinical manifestations of three groups of animals were observed after surgery, and brain tissue was taken for pathological examination 30 days later. Results: Brain histopathological examination: one rabbit in the experimental group showed inflammatory changes and no granuloma; no granuloma and inflammatory changes were observed in the negative control group; in the positive control group, granulomatous changes were observed in two rabbits and fibrillary glia astrocytosis in eight rabbits. Conclusion: Further studies are needed to establish an animal model by injecting eggs into the carotid artery and the method of injecting schistosoma egg suspension into brain tissue after skull drilling has a high success rate.
\end{abstract}

\section{Keywords}

Schistosoma japonicum, Granuloma of Worm Eggs, Animal Models, Mannitol

\section{Introduction}

Brain schistosomiasis was first reported by Yamagiwa in 1889, and came to widespread attention after the US invaded the Philippines in 1944 [1]. The eti- 
ology is mainly due to the granuloma formed by schistosoma eggs deposited in brain tissue, which causes headache, dizziness, blurred vision, incoordination, epilepsy and other neuropsychiatric symptoms [2]. The establishment of schistosoma animal model is very important for the study of schistosoma. Unfortunately, no animal model of the disease was reported for several decades after the discovery of the disease, and it was not until 1970 that the primate experiment of the disease was reported by John A. et al. [3]. Domestic scholars have successfully established the animal model of schistosoma granuloma in rabbits by injecting egg suspension into the brain tissue [4]. Some scholars opened the blood-brain barrier by radiotherapy and injected schistosoma eggs through the carotid artery; imaging studies showed successful modeling [5]. However, these methods have shortcomings. Injection after skull drilling directly destroys the blood-brain barrier, and radiotherapy also has a certain destructive effect, and can reduce animal immunity. To reduce these disadvantages, in this study, the animal model of brain schistosoma granuloma was further explored by injecting the egg suspension after opening the blood-brain barrier by injecting mannitol into carotid artery.

\section{Materials and Methods}

\subsection{Experimental Animals}

Thirty New Zealand white rabbits (weighing approximately $2 \mathrm{~kg}$, of either sex) were purchased from China Three Gorges University. They were kept in a $25^{\circ} \mathrm{C}$ animal house and fed standard feed and clean water.

\subsection{Egg Suspension and Injection Materials}

Live eggs of Schistosoma japonicum were provided by Jiangsu Institute of Schistosomiasis Control. After three times of washing with sterile normal saline, 5000 eggs $/ \mathrm{ml}$ suspension was prepared for experimental injection. There were some $1 \mathrm{ml}$ and $10 \mathrm{ml}$ syringes, indwelling trocars $(22 \mathrm{G})$.

\subsection{Animal Grouping and Processing}

Experimental group: ten rabbits were injected $1 \mathrm{ml}$ suspension of eggs. Positive control group: ten rabbits were injected $1 \mathrm{ml}$ suspension of eggs through their skulls. Negative control group: ten rabbits were injected $1 \mathrm{ml}$ suspension of eggs through carotid artery.

\subsection{Eggs Injection}

Experimental group: The rabbits were supine and fixed on the disinfection table, then their skin of the neck was ready for surgery. They were put under intravenous anesthesia with $3 \%$ sodium pentobarbital $(1 \mathrm{ml} / \mathrm{kg})$. Their neck skin was disinfected by tincture of iodine and covered with towel. The right common carotid artery was separated along the right side of the trachea. The indwelling trocar was inserted in a cephalic direction, then $20 \%$ mannitol was injected with 
a $10 \mathrm{ml}$ syringe from the other end of the trocar. Each rabbit was injected with 4 $\mathrm{ml} / \mathrm{Kg}$ in 30 seconds, then close the trocar. After $15 \mathrm{~min}, 1 \mathrm{ml}$ syringe was used to inject $1 \mathrm{ml}$ of prepared egg suspension through trocar, and $1 \mathrm{ml}$ sterile normal saline was injected to wash the trocar, then close the trocar. After $5 \mathrm{~min}$, the needle was pulled out and the puncture point was quickly compressed. After the bleeding stops, their skin was sewn up. The rabbits were returned to the animal room when the anesthetic effect disappeared.

Negative control group: The animals were injected with $1 \mathrm{ml}$ sterile saline instead of the egg suspension, and the rest of the steps were the same as the experimental group.

Positive control group: The rabbits were fixed in the prone position on the disinfection table, and their skin of head was ready for surgery. They were anesthetized by intravenous injection of $3 \%$ pentobarbital sodium $(1 \mathrm{ml} / \mathrm{kg})$ at the ear margin, then their skin of head was sterilized with iodine and then covered with towels. Incision was made along the midsagittal line of the top of the head, about $3 \mathrm{~cm}$ long. A hole was drilled by hand drill $0.5 \mathrm{~cm}$ behind the anterior fontanelle of the skull and $0.5 \mathrm{~cm}$ below the midline. The bleeding part of the skull was sealed with bone wax. A $1 \mathrm{ml}$ syringe was punctured into the dura mater at a depth of $0.5 \mathrm{~cm}$ vertically and then $1 \mathrm{ml}$ egg suspension was injected slowly. The skull was filled with gel foam and the scalp was sutured. The rabbits were returned to the animal room after the anesthetic effect had disappeared.

\subsection{Brain Histopathology}

Leica pathology biopsy machine, Olympus microscope and HE dye were all provided by the pathology department of Jingzhou First People's Hospital. Thirty days after the operation, the three groups of experimental animals were killed by intravenous injection of air through the ear margin. Their brain tissue was removed intact and preserved in $4 \%$ formalin solution. The brain tissue was fixed and dehydrated, embedded in paraffin block. The paraffin blocks were cut into thin slices which were then stained with HE dye. Two pathologists independently observed the existence of pathological changes such as egg granuloma, fibroglial cell healing and inflammation under the light microscope.

\section{Results}

\subsection{Clinical Manifestations}

After the operation, five rabbits of the experimental group showed anorexia and burnout, among which three showed unilateral limb movement disorder. The three rabbits were relieved two days later without epilepsy symptoms. In the positive control group, there were six animals with seizures, which were relieved after one week. No obvious abnormality was observed in the negative control group.

\subsection{Pathological Examination}

None of the three groups of experimental animals died. Pathological examina- 
tion of the negative control group showed no abnormality (Figure 1(a)). In the experimental group, inflammation was observed in the brain tissue of one rabbit (Figure $1(b)$ ) and the other nine rabbits had no obvious abnormality (Figure $1(\mathrm{c})$ ). In the positive control group, schistosoma egg antigen was interwoven with collagen fibers in the brain histopathological sections of 2 rabbits, with a few lymphocytes, neutrophils and eosinophils around (Figure 1(d) and Figure $1(\mathrm{e})$ ) and fibrillary glia was observed in the other 8 rabbits (Figure 1(f)).

\section{Discussion}

Schistosomiasis is one of the most prevalent parasitic diseases [6]. About 240 million people worldwide are infected with schistosoma and 700 million are at potential risk [7]. Schistosomiasis mainly includes schistosomiasis mansoni, schistosomiasis japonicum and schistosomiasis haematobium. Schistosoma mansoni and Schistosoma japonicum migrate to the distal mesenteric vein to lay eggs which pass through the intestinal wall and are eventually excreted in feces, while Schistosoma haematobium mainly migrates to the bladder vein [8]. The main pathogenesis of schistosomiasis is inflammatory granulomas caused by egg deposition in the liver and intestines of mammals [9]. Schistosoma eggs can also be deposited in brain, spinal cord, kidney, lung, breast, uterus, cervix, skin, bone marrow and other tissues [8]. Schistosomiasis of the central nervous system occurs as a result of abnormal adult migration or eggs deposition into the central nervous system [10]. Schistosoma mansoni and Schistosoma haematobium infections are more likely to cause spinal cord injuries, while schistosoma

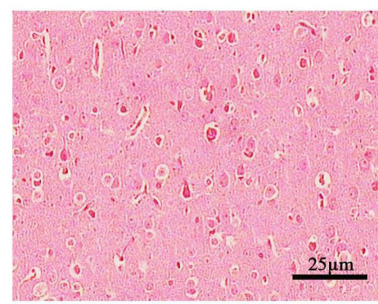

(a)

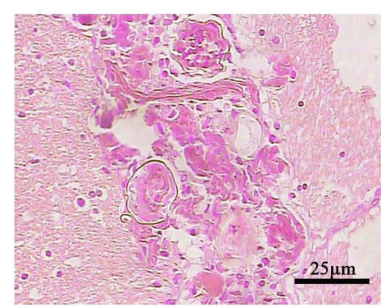

(d)

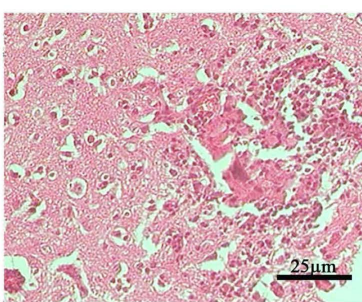

(b)

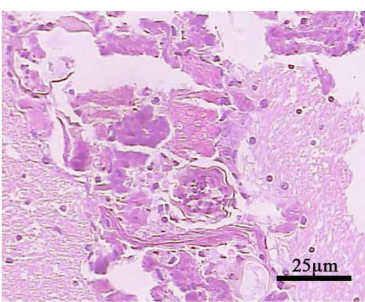

(e)

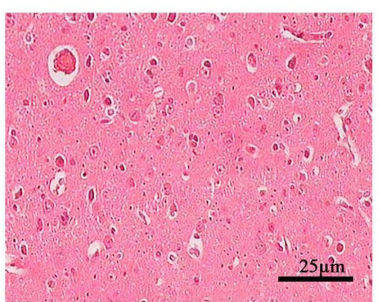

(c)

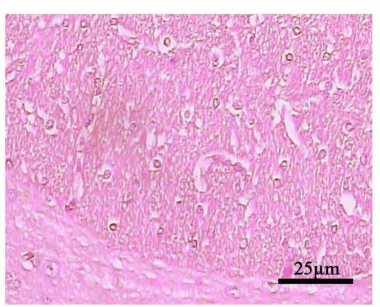

(f)

Figure 1. The pathological findings of the three groups of animals (H \& E stain, 200x). Pathological examination of negative control group showed no abnormality (a); In the experimental group, inflammation was observed in only one brain tissue (b) and the other nine rabbits had no obvious abnormality (c); In the positive control group, schistosoma egg antigen was interwoven with collagen fibers in the brain histopathological sections of 2 rabbits, with a few lymphocytes, neutrophils and eosinophils around ((d), (e)) and fibrillary glia was observed in the other 8 rabbits ( $f$ ). 
japonicum infection mainly causes brain damages [11]. There are two possible pathogenesis mechanisms of schistosomiasis in the central nervous system. First, schistosoma eggs pass through the blood circulation, from mesenteric capillaries to cerebral vessels, and then deposit, resulting in granuloma (also known as indirect deposition hypothesis). Second, adult schistosoma worms can lay eggs directly in the cerebrovascular system and cause granuloma (also known as direct deposition hypothesis) [2] [12].

JOHN A. was the first researcher to report an animal model of cerebral schistosomiasis [3]. In the 1970s, he experimented with schistosomiasis in black long-tailed monkeys, mainly by injecting hermaphrodite adults or eggs through the carotid artery and jugular vein. Granulomatous changes were found in brain tissue in only one monkey, which was injected twice with large numbers of schistosoma eggs through the carotid artery. The injection of massive schistosoma eggs into the carotid artery is likely to cause cerebral microvascular embolism, which can be proved by the observation of cerebral hemorrhage and schistosoma egg embolus by pathological examination. Therefore, his method has high animal mortality and low modeling success. Aloe et al. reported in 1996 that cercariae suspension was used to infect mice by contacting their skin, and then the mice were sacrificed in different periods [13]. Liver and brain tissues were taken at autopsy for pathological examination. Schistosoma eggs and granulomas were observed in the brain tissues of mice 15 - 20 weeks after infection of cercariae. In a preliminary study published in 2014, José Roberto Lambertucci reported for the first time that changes of schistosoma egg granulomas were observed in brain tissue of mice with neurological symptoms at 4 and 8 months after cercaria infection [14]. Luciana M Silva said no granulomatous changes were found in the brain tissue of mice infected with cercariae [15]. Therefore, whether this animal model can be used in schistosomiasis induced granuloma is still controversial. In 2011, wang peng, a domestic scholar, reported for the first time that the animal model of schistosoma egg granuloma was successfully established by injecting schistosoma eggs into the brain after drilling the skullsg of rabbits [4]. Subsequently, Xu Jia thinned the skulls of rabbits and injected the suspension of schistosoma eggs, which has also been successful [16]. According to Wang Peng's method, Ge Yuxi injected live schistosoma eggs to build models and conducted imaging studies on the animals [17].

Although the granuloma of schistosoma eggs can be observed after injecting schistosoma eggs directly into brain tissue, this method cannot fully reflect the pathological evolution and immunological changes of schistosomiasis in the natural state. The design of our experiment is closer to the pathogenesis of schistosomiasis in the brain under natural conditions, in which the schistosoma eggs deposit in brain tissue through the blood-brain barrier and cause granulomatous changes. Ge Yuxi used radiotherapy to open the blood-brain barrier of rabbits, and then injected schistosoma eggs into the carotid artery. Because of the blood-brain barrier, his method did not detect the granuloma of schistosoma eggs on pathological examination of brain tissue [5]. It has been found that 
mannitol can improve the permeability of the blood-brain barrier [18] [19]. Therefore, in this study, the blood-brain barrier was rapidly opened by injecting mannitol into carotid artery in advance, and then schistosoma egg suspension was injected into the carotid artery in the hope that schistosoma eggs could more easily reach the brain through the blood-brain barrier. Compared with the drilling method, the destruction of the blood-brain barrier is reduced, and the influence on immunity is reduced compared with the radiotherapy. The trocar puncture injection can better ensure the puncture success rate and the suspension of injected eggs will not flow out of the puncture point due to the blood pressure. The brain tissues of all experimental animals were pathologically examined 30 days after the injection of eggs into the carotid artery. No abnormal changes were observed in the brain tissues of the negative control group animals. Inflammatory changes were found in one of the ten rabbits of the experimental group. However, no changes of granuloma and fibrillary glia were found in the experimental group, which were found in the positive control group. The reasons are as follows: 1) Schistosoma eggs do not enter the brain through the blood-brain barrier, but only some antigens secreted by the eggs reach the brain, thus forming inflammatory changes. 2) The number of eggs injected may be relatively low, most of which are directly collected in the cerebral veins through the cerebral arteriovenous pathway, and then reach other tissues and organs through the blood circulation. 3) The observation time after injection of the eggs may be short, and the eggs may be located in the capillaries of brain tissue.

\section{Conclusion}

This experimental method has made some achievements, but still has some shortcomings, and should be improved. Compared with the success rate, the injection of schistosomiasis eggs after drilling into the skull was higher than that by carotid artery injection, and the pathological changes were typical. The formation mechanism of cerebral schistosomiasis by injection of schistosoma eggs into carotid artery is close to that of natural state. However, the method still needs further research and improvement.

\section{Conflicts of Interest}

The authors declare no conflicts of interest regarding the publication of this paper.

\section{References}

[1] Mitsuno, T. (1955) Cerebral Granuloma Caused by Schistosoma japonicum. Journal of Neurosurgery, 12, 291-299. https://doi.org/10.3171/jns.1955.12.3.0291

[2] Scrimgeour, E.M. and Gajdusekm, D.C. (1985) Involvement of the Central Nervous System in Schistosoma mansoni and S. haematobium Infection. A Review. Brain, 108, 1023-1038. https://doi.org/10.1093/brain/108.4.1023

[3] Jane, J.A., Warren, K.S. and Van den Noort, S. (1970) Experimental Cerebral Schistosomiasis japonica in Primates. Journal of Neurology, Neurosurgery, and Psychia- 
try, 33, 426-430. https://doi.org/10.1136/jnnp.33.4.426

[4] Wang, P., et al. (2011) Establishment of a Cerebral Schistosomiasis Experimental Model in Rabbits. Neuroscience Bulletin, 27, 91-98.

https://doi.org/10.1007/s12264-011-1127-0

[5] Ge, Y.X., et al. (2017) Preliminary Study to Create an Animal Model of Cerebral Granuloma by Injecting Schistosoma japonicum Eggs through the Carotid Artery. Journal of Pathogen Biology, 12, 864-867+878.

[6] Zhang, Y., MacArthur, C., Mubila, L. and Baker, S. (2010) Control of Neglected Tropical Diseases Needs a Long-Term Commitment. BMC Medicine, 8, 67. https://doi.org/10.1186/1741-7015-8-67

[7] Wei, Y., et al. (2018) The Diagnosis and Treatment Introspection of the First Imported Case of Atypical Cerebral Schistosomiasis in Guangzhou City. PLOS Neglected Tropical Diseases, 12, e0006171. https://doi.org/10.1371/journal.pntd.0006171

[8] Carod-Artal, F.J. (2010) Neuroschistosomiasis. Expert Review of Anti-Infective Therapy, 8, 1307-1318. https://doi.org/10.1586/eri.10.111

[9] Dang-Trinh, M.A., et al. (2018) Utilization of Real Time PCR for the Assessment of Egg Burden in the Organs of Schistosoma japonicum Experimentally Infected Mice. Experimental Parasitology, 189, 61-65. https://doi.org/10.1016/j.exppara.2018.04.014

[10] Wen, S.C.H., et al. (2019) Pediatric Neuroschistosomiasis: A Case Report and Review of the Literature. Journal of the Pediatric Infectious Diseases Society, 8. https://doi.org/10.1093/jpids/piz009

[11] Lambertucci, J.R., Silva, L.C. and do Amaral, R.S. (2007) Guidelines for the Diagnosis and Treatment of Schistosomal Myeloradiculopathy. Revista da Sociedade Brasileira de Medicina Tropical, 40, 574-581. https://doi.org/10.1590/S0037-86822007000500016

[12] Wang, P., et al. (2010) Pathogenesis of Granuloma of Schistosomiasis of the Central Nervous System. Chinese Journal of Nervous and Mental Diseases, 36, 378-380.

[13] Aloe, L., Moroni, R., Fiore, M. and Angelucci, F. (1996) Chronic Parasite Infection in Mice Induces Brain Granulomas and Differentially Alters Brain Nerve Growth Factor Levels and Thermal Responses in Paws. Acta Neuropathologica, 92, 300-305. https://doi.org/10.1007/s004010050522

[14] Lambertucci, J.R., et al. (2014) Brain Schistosomiasis in Mice Experimentally Infected with Schistosoma mansoni. Revista da Sociedade Brasileira de Medicina Tropical, 47, 251-253. https://doi.org/10.1590/0037-8682-0083-2013

[15] Silva, L.M., Oliveira, C.N. and Andrade, Z.A. (2002) Experimental Neuroschistosomiasis: Inadequacy of the Murine Model. Memórias do Instituto Oswaldo Cruz, 97, 599-600. https://doi.org/10.1590/S0074-02762002000400028

[16] Xu, J., et al. (2013) Preliminary Study on Establishing an Animal Model of Neuroschistosomiasis by Direct Injection of Schistosoma japonicum Eggs through Skull. Chinese Journal of Schistosomiasis Control, 25, 28-30.

[17] Ge, Y.X., et al. (2017) MRI Findings of Cerebral Schistosomiasis in Acute Stage Establishment of Experimental Model of Acute Cerebral Schistosomiasis with Rabbits. Chinese Journal of Schistosomiasis Control, 29, 554-558.

[18] Pan, G.Y., Liu, X.D. and Liu, G.Q. (2000) Intracarotid Infusion of Hypertonic Mannitol Changes Permeability of Blood-Brain Barrier to Methotrexate in Rats. Acta Pharmacologica Sinica, 21, 613-616. 
[19] Brown, R.C., Egleton, R.D. and Davis, T.P. (2004) Mannitol Opening of the Blood-Brain Barrier: Regional Variation in the Permeability of Sucrose, But Not 86Rb+ or Albumin. Brain Research, 1014, 221-227.

https://doi.org/10.1016/j.brainres.2004.04.034 